\title{
Counter-rotating Kerr manifolds separated by a fluid shell
}

\author{
J P Krisch and E N Glass \\ Department of Physics, University of Michigan, Ann Arbor, MI 48109, USA \\ E-mail: englass@umich.edu
}

Received 3 April 2009, in final form 1 July 2009

Published 12 August 2009

Online at stacks.iop.org/CQG/26/175010

\begin{abstract}
We describe a spheroidal fluid shell between two Kerr vacuum regions which have opposite rotation parameters. The shell has a stiff equation of state and a heat flow vector related to the rotational Killing current. The shell description is useful in exploring the significance of counter-rotation in Kerr metric matches.
\end{abstract}

PACS number: $04.20 . \mathrm{CV}$

\section{Introduction}

The Kerr vacuum metric is believed to describe the spacetime exterior to compact rotating objects such as neutron stars [1]. Kerr and vacuum Schwarzschild are considered the two most fundamental explicit solutions of general relativity. While there are a variety of interior fluid models that provide the source of the Schwarzschild mass parameter, a single interior fluid region matched to the Kerr vacuum has not yet been discovered. Some of the early investigations looked at models expressed as an expansion of the Kerr angular momentum parameter ' $a$ '. Brill and Cohen [2] showed that to $O(a)$, a rotating spherical shell bounding a flat interior could be matched to Kerr. DeLaCruz and Israel [3] extended the model to order $O\left(a^{2}\right)$. Hartle and Thorne [4] studied slowly rotating relativistic stars and found that, with their general metric, any 'slowly rotating configuration' could match vacuum Kerr up to second order in rotational speed, a result that Cohen [5] noticed for $O(a)$ perfect fluids. McCrea [6] and Florides [7, 8], using a smallness parameter ' $k$ ', discussed spherical and spheroidal matches to $O(k)$ and $O\left(k^{5}\right)$, respectively.

One of the technical problems with matching the Kerr metric to an interior is duplicating the dependence on $\cos ^{2} \theta$, found in all of the Kerr metric functions. The strategy of keeping the angular behaviour and replacing some or all of the functions of the radial coordinate with unknown functions has been discussed by Krasinski [9]. Attempts to simplify the general match with a perfect fluid interior have been unsuccessful $[9,10]$.

Because some interesting astrophysical objects have spherical topology, there are many treatments matching Kerr to matter distributions with spheroidal boundaries. The most common boundary is $r=R_{0}$, which in the Kerr geometry is spheroidal. Other choices 
are Hogan's [11] interior solution with boundary $r^{2}-b r+a^{2} \cos ^{2} \theta=0$, and the GursesGursey [12] spheroid, $r_{0}^{2}\left(x^{2}+y^{2}+z^{2}\right)-a^{2} z^{2}=r_{0}^{2}\left(r_{0}^{2}+a^{2}\right)$. Both models develop unphysical stress-energy in the interior [13].

For models with spherical topology, one has to make a choice of a metric interior, and often a choice of the stress-energy form. Disc models avoid these choices by treating the disc as a boundary between two Kerr manifolds, and then use the Israel [14] method to create a disc with stress-energy determined by the extrinsic curvature jump across the disc. Discs are useful models for flat galaxy-like objects with diffuse edges, and the disc structure can be motivated by the shape of the Kerr ring singularity. Israel's [15] source for Kerr was a layer of mass in an equatorial disc. The disc had negative density and spun superluminally. Lopez [19] studied rigidly rotating discs; a negative mass disc with a line of infinite positive masses along the edges was described by Keres [18]. Hamity's [16] disc model has no density and positive stress. Hamity and Lamberti [17] found that these disc models violated the strong and weak energy conditions, as reflected in their mass description. McManus [10] combined a toroidal shell with a disc using both positive and negative mass and angular momentum. Following a treatment of counter-rotating sources for static vacuum solutions [21], Bičák and Ledvinka [22] described a disc source made of two counter-rotating particle currents; these counter-rotating current models appear to solve many of the matter problems occurring in the papers reviewed by Hamity and Lamberti [17]. Counter-rotation was also considered in an early disc model by Morgan and Morgan [20]. One of their examples, using a gravitational potential communicated by Bardeen, was a disc of uniformly counter-rotating dust. While they made no connection to Kerr, the appearance of counter-rotation in a model with physical matter is interesting. Counter-rotation has also appeared in the models of Marek and Haggag [24, 25] and Ramadan [26], who described Kerr matches with a rotating thick matter shell around a static matter core with counter-rotation occurring in the thick shell.

A component of the disc description which has produced interesting results is counterrotation and, for disc matches, vacuum Kerr on both sides of the matching mass distribution. Using Kerr on both sides of a matching disc involves a change in the sense of $z$-coordinate increase (in cylindrical coordinates). For example, the disc match of Bičák and Ledvinka [22] has outward-directed normals on either side of the disc. The flip in the direction of a positive normal crossing the disc produces a non-zero jump in the extrinsic curvature for the same metric. For spheroidal shells outside of the event horizons, the interior and exterior normals are both outward pointing, but an analogous choice is opposite senses for the azimuthal angle. For a spheroidal shell with Kerr on both sides, this choice is metrically equivalent to choosing a positive angular momentum parameter in the exterior region and a negative parameter in the interior. Developing a spheroidal model containing some of the best elements of the disc models, counter-rotation and Kerr on both sides of the boundary, will allow the importance of these features to be investigated for other Kerr matches.

In this paper, we describe a spheroidal shell between two vacuum Kerr metrics where the 2-metrics differ only in the sign of the parameter ' $a$ '. The shell is located outside of the event horizon. In the following section, we describe the metric of the shell and the observers who will interpret its stress-energy. Using the Israel [14] formalism, the shell stress-energy is developed in section 3. We find that the shell has a stiff equation of state (not imposed), with a heat flow component following from the Israel stress condition, and which is necessary to provide a positive angular momentum parameter at infinity. The angular speed of the shell is discussed in the fourth part of the paper and a simple model is also discussed. The shell is potentially useful as a tool to explore the effects of counter-rotation in Kerr matches. It also provides a simple model with which to describe some general relativistic effects in astrophysical objects with shell structure [27-30]. 


\section{Metric and observers}

The Boyer-Lindquist (BL) form of vacuum Kerr for the interior (-) and exterior (+) is

$$
\begin{aligned}
& \mathrm{d} s_{ \pm}^{2}=-f \mathrm{~d} t^{2}-2 k_{ \pm} \mathrm{d} t \mathrm{~d} \varphi+(\Sigma / \Delta) \mathrm{d} r^{2}+\Sigma \mathrm{d} \vartheta^{2}+l \mathrm{~d} \varphi^{2} \\
& \Sigma=r^{2}+a^{2} \cos ^{2} \vartheta, \quad \Delta=r^{2}+a^{2}-2 m r, \\
& f=1-2 m r / \Sigma, \\
& k_{ \pm}= \pm 2 m a r \Sigma^{-1} \sin ^{2} \vartheta, \\
& l=\left[\left(r^{2}+a^{2}\right)^{2}-a^{2} \Delta \sin ^{2} \vartheta\right] \Sigma^{-1} \sin ^{2} \vartheta, \\
& D^{2}=f l+k_{ \pm}^{2}=\Delta \sin ^{2} \vartheta .
\end{aligned}
$$

The observers on either side of a matching Israel layer [14] should agree on the induced layer metric. For two Kerr vacua, the observers who agree on a metric form are the zero angular momentum observers (ZAMOs), moving with 4-velocity

$$
U_{ \pm}^{i}=\sqrt{l / D^{2}}\left[1,0,0, \Omega_{ \pm}\right], \quad \Omega_{ \pm}=k_{ \pm} / l .
$$

The metric for the ZAMOs is

$$
\mathrm{d} h^{2}=-\left(D^{2} / l\right) \mathrm{d} t^{2}+(\Sigma / \Delta) \mathrm{d} r^{2}+\Sigma \mathrm{d} \vartheta^{2}+l \mathrm{~d} \psi_{ \pm}^{2}
$$

where their angular coordinate $\psi_{ \pm}$is related to the $\operatorname{BL} \varphi$ by

$$
\mathrm{d} \psi_{ \pm}=\mathrm{d} \varphi-\Omega_{ \pm} \mathrm{d} t .
$$

The boundary surface between the two metrics is the spheroidal surface defined by $r=R_{0}$ with normal vector

$$
n_{i}=[0, \sqrt{\Sigma / \Delta}, 0,0] .
$$

The two ZAMOs agree on the shell metric equation (3), with the jump in the extrinsic curvature providing the shell stress-energy [14].

\section{Stress-energy}

The Israel stress-energy [14], $S_{i j}$, is formed from jumps in the extrinsic curvature, $K_{i j}$, with

$$
-8 \pi S_{i j}=\left\langle K_{i j}\right\rangle-\frac{1}{2} h_{i j}\left\langle K_{k}^{k}\right\rangle
$$

and extrinsic curvature

$$
K_{i j}=n_{a ; b} e_{i}^{a} e_{j}^{b} .
$$

$e_{i}^{a}$ are the tangents to the layer. The extrinsic curvature components are, with $\digamma=$ $\sqrt{\Delta} /(2 \sqrt{\Sigma})$,

$$
\begin{aligned}
& K_{t t}^{ \pm}=-\digamma\left[\partial_{r} f+2 \Omega_{ \pm} \partial_{r} k_{ \pm}-\Omega_{ \pm}^{2} \partial_{r} l\right] \\
& K_{\vartheta \vartheta}^{ \pm}=\digamma \partial_{r} \Sigma \\
& K_{\psi \psi}^{ \pm}=\digamma \partial_{r} l \\
& K_{t \psi}^{ \pm}=-\digamma\left[\partial_{r} k_{ \pm}-\Omega_{ \pm} \partial_{r} l\right]=-l \digamma \partial_{r}\left(k_{ \pm} / l\right) .
\end{aligned}
$$


Only the component $K_{t \psi}$, which is odd in ' $a$ ', will contribute to the jump:

$$
\begin{aligned}
& \left\langle K_{i}^{i}\right\rangle=0 \\
& \left\langle K_{t \psi}\right\rangle=l \digamma\left(\partial_{r} \Omega_{-}-\partial_{r} \Omega_{+}\right)
\end{aligned}
$$

with $\Omega_{-}=-\Omega_{+}$

$$
\left\langle K_{t \psi}\right\rangle=-2 l \digamma \partial_{r} \Omega_{+} .
$$

The associated stress-energy is

$$
8 \pi S_{t \psi}=-\left\langle K_{t \psi}\right\rangle=2 l \digamma \partial_{r} \Omega_{+} .
$$

This stress-energy can be analyzed using an anisotropic fluid with heat flow, $q_{i}=\left[q_{t}, 0,0, q_{\psi}\right]$ :

$$
8 \pi S_{i j}=\sigma V_{i} V_{j}+P_{(\psi)} \Psi_{i} \Psi_{j}+q_{i} V_{j}+q_{j} V_{i}
$$

where $\Psi^{i}$ is the unit vector for the azimuthal coordinate and $V^{i}$ is the unit velocity of the layer:

$$
\begin{aligned}
& V_{ \pm}^{i}=l^{-1 / 2}\left[(D / l)^{2}-\left(\omega_{ \pm}-\Omega_{ \pm}\right)^{2}\right]^{-1 / 2}\left[1,0,0, \omega_{ \pm}-\Omega_{ \pm}\right] \\
& \Psi_{ \pm}^{i}=\left(l^{1 / 2} / D\right)\left[(D / l)^{2}-\left(\omega_{ \pm}-\Omega_{ \pm}\right)^{2}\right]^{-1 / 2}\left[\left(\omega_{ \pm}-\Omega_{ \pm}\right), 0,0,(D / l)^{2}\right]
\end{aligned}
$$

with $\omega_{ \pm}=\mathrm{d} \varphi / \mathrm{d} t$ the exterior/interior rotational speed for the layer with BL angle $\varphi$. Different $\omega_{ \pm}$on either side of the layer allows models that will include counter-rotation in the layer motion. The density, pressures and heat flow arising from the stress-energy are

$$
\begin{aligned}
& \sigma_{ \pm}=P_{(\psi) \pm}=\frac{2\left(\omega_{ \pm}-\Omega_{ \pm}\right)}{(D / l)^{2}-\left(\omega_{ \pm}-\Omega_{ \pm}\right)^{2}} \sqrt{\frac{\Delta}{\Sigma}} \partial_{r} \Omega_{+} \\
& P_{(\vartheta)}=0 \\
& q_{t \pm}=-\left(\omega_{ \pm}-\Omega_{ \pm}\right) q_{\psi_{ \pm}} \\
& q_{i \pm} \Psi_{ \pm}^{i}=-\sqrt{\frac{\Delta}{\Sigma}}\left[\frac{l \partial_{r} \Omega_{+}}{D}\right] \frac{(D / l)^{2}+\left(\omega_{ \pm}-\Omega_{ \pm}\right)^{2}}{(D / l)^{2}-\left(\omega_{ \pm}-\Omega_{ \pm}\right)^{2}}
\end{aligned}
$$

All stress-energy parameters are evaluated at a shell radius exterior to the outer event horizon, $R_{0}>m+\sqrt{a^{2}-m^{2}}$. Note that the stiff equation of state is not imposed but arises from the stress-energy content.

\section{Rotational speed}

The derivative of the exterior ZAMO angular speed is negative: $\partial_{r} \Omega_{+}<0$ for $R_{0}$ outside of the first event horizon at $\Delta=0$. In the exterior $\omega_{+}<k_{+} / l$ is necessary for positive density. This is a smaller value than the maximum allowed rotational speed for an exterior Kerr observer [31], $\left(\omega_{+}\right)_{\max }=k_{+} / l+D / l$. A stricter condition is set on $\omega_{-}$. For positive density, $\omega_{-}$ must be negative and with size larger than $\left|k_{+} / l\right|$. The Kerr constraint bounds $\omega_{-}$such that $\left(\omega_{-}\right)_{\min }=-\left(k_{+} / l+D / l\right)$. The conditions on $\omega_{ \pm}$for positive density with the Kerr constraints are

$$
\begin{aligned}
& k_{+} / l-D / l \leqslant \omega_{+}<k_{+} / l \\
& \omega_{-}<0 \\
& k_{+} / l<\left|\omega_{-}\right| \leqslant k_{+} / l+D / l .
\end{aligned}
$$


$\omega_{+}$can be positive or negative, with counter-rotation developing if it is positive. One can find a relation between $\omega_{+}$and $\omega_{-}$by considering density relations. Equating the densities, $\sigma_{+}=\sigma_{-}$, we have

$$
\frac{\left(\omega_{+}-\Omega_{+}\right)}{D^{2}-l^{2}\left(\omega_{+}-\Omega_{+}\right)^{2}}=\frac{\left(\omega_{-}-\Omega_{-}\right)}{D^{2}-l^{2}\left(\omega_{-}-\Omega_{-}\right)^{2}} .
$$

Defining a scaled angular speed $\bar{\omega}_{ \pm}=\omega_{ \pm} / \Omega_{+}$, this can be written as

$$
D^{2}\left(\bar{\omega}_{+}-\bar{\omega}_{-}-2\right)=k_{+}^{2}\left(\bar{\omega}_{+}-1\right)\left(\bar{\omega}_{-}+1\right)\left(\bar{\omega}_{-}-\bar{\omega}_{+}+2\right) .
$$

The two possible conditions on $\bar{\omega}_{ \pm}$are

$$
\bar{\omega}_{+}=\bar{\omega}_{-}+2, \quad\left(1-\bar{\omega}_{+}\right)\left(1+\bar{\omega}_{-}\right)=D^{2} / k_{+}^{2}
$$

The first condition establishes a relation between the two rotational speeds. It is not possible to satisfy the second condition for positive density layers. In the region

$$
0<\bar{\omega}_{+}<1, \quad 1<\left|\bar{\omega}_{-}\right|<2,
$$

the layer is observed to rotate in the same direction as the corresponding counter-rotating ZAMO. The functional form of $\omega_{ \pm}$is not determined, but some convenient choices can be made to explore the density. For example, the structure of the density suggests a simplifying choice:

$$
\omega_{+}-\Omega_{+}=-n(D / l) \Sigma^{\gamma} \sin \theta, \quad \bar{\omega}_{+}=1-n \frac{D \Sigma^{\gamma} \sin \theta}{k}=1-n \frac{\Sigma^{\gamma+1} \sqrt{\Delta}}{2 m a r} .
$$

The factor $\Sigma^{\gamma}$ is added to facilitate a discussion (in the last part of the paper) of the shell behaviour as the ring singularity is approached. A constraint on the size of the shell is set as a function of the model parameters:

$$
0<n \frac{\Sigma^{\gamma+1} \sqrt{\Delta}}{2 m a r}<1
$$

The density is

$$
\sigma_{+}=n \frac{4 m a \Sigma^{\gamma-3 / 2} \sin ^{2} \theta\left[3 r^{4}+r^{2} a^{2}\left(2-\sin ^{2} \theta\right)-a^{4} \cos ^{2} \theta\right]}{\left[\left(r^{2}+a^{2}\right)^{2}-a^{2} \Delta \sin ^{2} \theta\right]\left(1-n^{2} \Sigma^{2 \gamma} \sin ^{2} \theta\right)}
$$

at the equator $\theta=\pi / 2$ and $\Sigma=r^{2}$

$$
\left(\sigma_{+}\right)_{\text {equator }}=n \frac{4 m a r^{2 \gamma-2}\left(3 r^{2}+a^{2}\right)}{\left(r^{3}+a^{2} r+2 m a^{2}\right)\left(1-n^{2} r^{4 \gamma}\right)} .
$$

The equatorial density is a smoothly increasing function of $r$ coming in towards the first event horizon $(\Delta=0)$. As a function of the polar angle, the density is peaked around the equator, decreasing to zero at the poles. To an exterior observer, the mass distribution is suggestive of the ring singularity. However, with opposite rotation parameters in the interior and exterior metrics, the shell is a source of angular momentum and mass in addition to the ring contributions. Using the same form for the rotational speed, the size of the heat flow vector and its components are

$$
\begin{aligned}
& q=\sqrt{q_{i} q^{i}}=q_{i \pm} \Psi_{ \pm}^{i} \\
& q_{i \pm} \Psi_{ \pm}^{i}=\frac{2 m a \sin \theta}{\Sigma^{3 / 2}}\left[\frac{3 r^{4}+r^{2} a^{2}\left(2-\sin ^{2} \theta\right)-a^{4} \cos ^{2} \theta}{\left(r^{2}+a^{2}\right)^{2}-a^{2} \Delta \sin ^{2} \theta}\right]\left(\frac{1+n^{2} \Sigma^{2 \gamma} \sin ^{2} \theta}{1-n^{2} \Sigma^{2 \gamma} \sin ^{2} \theta}\right) \\
& q_{t}=n(D / l)\left(\Sigma^{\gamma} \sin \theta\right) q_{\psi_{ \pm}} \quad q_{\psi_{ \pm}}=\frac{q_{i \pm} \Psi_{ \pm}^{i} \sqrt{l}}{\left[1-n^{2} \Sigma^{2 \gamma} \sin ^{2} \theta\right]^{1 / 2}} .
\end{aligned}
$$


The heat current in the shell, like the density, is peaked around the equator as one would expect from a rotating mass distribution. The shell is a source of angular momentum but not the singular ring usually associated with vacuum Kerr. The shell surrounds a spinning source whose angular momentum parameter is opposite to the one observed at infinity. The Komar angular momentum of the shell, calculated in the appendix, is $2 m a$. The shell current angular momentum, combined with the counter-rotating ring angular momentum, provides the $+a$ observed at infinity.

\section{Discussion}

There are parallels between the Kerr rotating disc [22] and the Kerr rotating shell. The disc is described with two sets of observers. The $\varphi$-isotropic observers (FIOs) see a diagonal stressenergy tensor and interpret the matter content as two streams of particles counter-rotating with the same speed. These observers are rotating with respect to the disc ZAMOs who see the streams counter-rotate with different speeds. The equation of state of the disc depends on the rotational speed seen by the FIOs.

The observer sets for the Kerr shell are the ZAMOs who see a diagonal metric with the angular coordinate $\psi_{ \pm}$, and the observers who use the BL coordinate $\varphi$. The ZAMOs are counter-rotating with respect to each other. The \pm shell ZAMOs who agree on the size of the layer density set limits on $\omega_{ \pm}$. Within these limits, the BL observers can interpret the shell as having two counter-rotating elements or not, depending on the sign of $\omega_{+}$. The Kerr shell has a richer set of motions than the Kerr disc but a very limited equation of state. The shell contains a $P=\sigma$ fluid while the content of the Kerr disc ranges from dust to stiff matter. The Kerr shell has heat flow, while the Kerr disc does not.

The heat flow vector provides an interesting insight. If $\omega_{ \pm}=\Omega_{ \pm}$, the density, pressure and timelike component of the heat flow vanish. The stress-energy becomes

$$
8 \pi S_{i j}=q_{i} N_{j}+N_{i} q_{j}
$$

with $V^{i} \rightarrow N^{i}=\left[l^{1 / 2} / D, 0,0,0\right]$ timelike. A spatial heat component remains

$$
q_{\psi+}=-\frac{l^{3 / 2}}{D} \sqrt{\frac{\Delta}{\Sigma}} \partial_{r} \Omega_{+} .
$$

This heat component can be identified with the rotational Killing current [32]. Rewriting it in terms of the $\psi$ Killing vector $\xi_{(\psi)}^{i}$, we have

$$
q_{\psi+}=-8 \pi S_{i j} N^{i} \xi_{(\psi)}^{j},
$$

which is the integrand of a Komar angular momentum calculation (see appendix A). The Komar mass and angular momentum of the disc and the shell are conceptually very different. The disc is the source of the metric on either side, and the Kerr mass and angular momentum parameters are usually referred to the disc. The shell surrounds a spinning source whose angular momentum parameter is opposite to that observed at infinity. The Komar mass of the shell is zero and the angular momentum is $2 m a$. Other examples with zero Komar mass and non-zero Komar angular momentum have been discussed by Glass and Krisch [33]. These Komar results motivate the same Schwarzschild mass parameter in the interior and exterior as well as conservation of angular momentum over the whole region and reflect the result of Ansorg and Petroff [34, 35] for Komar mass; that Komar quantities seem more closely related to an object in a gravitational environment than to properties of the source by itself.

While physical observers lie outside the first Kerr horizon, it is of interest to ask about the shell behaviour as $r$ approaches the ring singularity at $R_{0}=0$. Balasin and Nachbagauer [36] 
have developed a distributional method for exhibiting the ring structure of the Kerr source. Using outgoing null coordinates $[\chi, v]$ with the definition $\mathrm{d} \psi=\mathrm{d} \chi+(k / l) \mathrm{d} v$ allows a view inward towards the ring singularity, where the distributional support for the ring has been established. The functional form for the density and heat flow are the same as in equation (27). The renormalization factor $\Sigma^{\gamma}$ with $\gamma=1$ allows a non-zero density similar to the toroidal density discussed by McManus [10]. The equatorial heat current is singular. In this limit, the heat flow current overlies the ring singularity with the net angular momentum parameter $+a$, and the manifold is a single Kerr spacetime.

The shell model developed in this paper, along with the Kerr disc models, provides a model set that can be used to study some of the challenges of a general Kerr match. It incorporates some features which have been useful in disc models, such as counter-rotation and the same metric structure on each side of the shell. Differences such as a stiff equation of state and heat flow are suggestive of directions for further study in more detailed models. The model could also be useful in describing some general relativistic aspects of sources with a shell structure [27-30].

\section{Appendix. Komar mass and angular momentum}

The shell that separates the two vacuum Kerr regions has a physical density, stress and a stiff fluid equation of state. The Komar mass and angular momentum can be calculated for the layer as seen by BL observers where the integrals are over a $t=$ constant hypersurface $\Pi$ with $N^{a}$ the normal to $\Pi$.

Asymptotically, as the spheroidal boundary is taken to infinity, the normal is

$$
\lim _{R_{0} \rightarrow \infty} N^{a}=\delta_{(t)}^{a}
$$

Using the coordinate transformation, equation (4), to evaluate the BL stress-energy yields

$$
\begin{aligned}
& 8 \pi S_{(t)(t)}^{\mathrm{BL}}=-2 \Omega_{+} S_{(t)(\psi)}^{\mathrm{BL}}=-2 k_{+} \sqrt{\frac{\Delta}{\Sigma}} \partial_{r} \Omega_{+}, \\
& 8 \pi S_{(t)(\varphi)}^{\mathrm{BL}}=S_{(t)(\psi)}^{\mathrm{BL}}=\sqrt{\frac{\Delta}{\Sigma}} l \partial_{r} \Omega_{+}, \\
& \operatorname{trace} S^{\mathrm{BL}}=0 .
\end{aligned}
$$

Asymptotically we have

$$
\begin{aligned}
& \partial_{r} \Omega_{+} \sim-\frac{6 m a}{r^{4}} \\
& 8 \pi S_{(t)(t)}^{\mathrm{BL}} \sim \delta\left(r-R_{0}\right) \frac{4 m a \sin ^{2} \vartheta}{r} \frac{6 m a}{r^{4}} \\
& \sqrt{-g} d^{3} x \sim r^{2} \sin \vartheta \mathrm{d} r \mathrm{~d} \vartheta \mathrm{d} \varphi
\end{aligned}
$$

The Komar mass is

$$
\begin{aligned}
M_{k \text {-layer }} & =2 \lim _{R_{0} \rightarrow \infty} \int_{\Pi} S_{a b}^{\mathrm{BL}} N^{a} \delta_{(t)}^{b} \sqrt{-g} \mathrm{~d}^{3} x \\
& =2 \lim _{R_{0} \rightarrow \infty} \int_{\Pi} S_{(t)(t)}^{\mathrm{BL}} \sqrt{-g} \mathrm{~d}^{3} x \\
& =2 \lim _{R_{0} \rightarrow \infty} 2 \pi \int_{\Pi} \delta\left(r-R_{0}\right) \frac{4 m a \sin ^{2} \vartheta}{8 \pi r} \frac{6 m a}{r^{4}} r^{2} \sin \vartheta \mathrm{d} r \mathrm{~d} \vartheta
\end{aligned}
$$




$$
\begin{aligned}
& =2 \lim _{R_{0} \rightarrow \infty} \int_{\Pi} \frac{m a \sin ^{3} \vartheta}{R_{0}} \frac{6 m a}{R_{0}^{4}} R_{0}^{2} \mathrm{~d} \vartheta \\
& =0 .
\end{aligned}
$$

The Komar angular momentum is

$$
\begin{aligned}
L & =-\lim _{R_{0} \rightarrow \infty} \int_{\Pi} S_{a b} N^{a} \delta_{(\varphi)}^{b} \sqrt{-g} \mathrm{~d}^{3} x \\
& =-\lim _{R_{0} \rightarrow \infty} \int_{\Pi} S_{(t)(\phi)} \sqrt{-g} \mathrm{~d}^{3} x \\
& =-\lim _{R_{0} \rightarrow \infty} \int_{\Pi}-\delta\left(r-R_{0}\right) \frac{6 m a r^{2} \sin ^{2} \vartheta}{8 \pi r^{4}} 2 \pi r^{2} \sin \vartheta \mathrm{d} r \mathrm{~d} \vartheta \\
& =\lim _{R_{0} \rightarrow \infty} \int_{\Pi} \frac{6 m a R_{0}^{2} \sin ^{2} \vartheta}{4 R_{0}^{4}} R_{0}^{2} \sin \vartheta \mathrm{d} \vartheta \\
& =2 m a .
\end{aligned}
$$

\section{References}

[1] Bonnor W B 1992 Physical interpretations of vacuum solutions of Einstein's equations Gen. Rel. Grav. 24551

[2] Brill D R and Cohen J M 1966 Rotating masses and their effect on inertial frames Phys. Rev. 1431011

[3] DeLaCruz V and Israel W 1968 Spinning shell as a source of the Kerr metric Phys. Rev. 1701187

[4] Hartle J B and Thorne K S 1968 Slowly rotating relativistic stars: II. Models for neutron stars and supermassive stars Astrophys. J. 153807

[5] Cohen J M 1967 Note on the Kerr metric and rotating masses J. Math. Phys. 81477

[6] McCrea J D 1973 Gravitational field of a uniformly rotating sphere in third approximation Proc. R. Irish Acad. A 7325

[7] Florides P S 1973 A rotating sphere as a possible source of the Kerr metric Nuovo Cimento B 131

[8] Florides P S 1975 A rotating spheroid as a possible source of the Kerr metric Nuovo Cimento B 25251

[9] Krasinski A 1978 Ellipsoidal space-times, sources for the Kerr metric Ann. Phys., NY 11222

[10] McManus D 1991 A toroidal source for the Kerr black hole geometry Class. Quantum Grav. 8863

[11] Hogan P A 1976 An interior Kerr solution Lett. Nuovo Cimento 1633

[12] Gurses M and Gursey F 1975 Lorentz covariant treatment of the Kerr-Schild geometry J. Math. Phys. 162385

[13] Collas P 1978 Simple tests for proposed interior Kerr metrics Lett. Nuovo Cimento 2168

[14] Israel W 1966 Singular hypersurfaces and thin shells in general relativity Nuovo Cimento B 441

[15] Israel W 1970 Source of the Kerr metric Phys. Rev. D 2641

[16] Hamity V 1976 An 'interior' of the Kerr metric Phys. Lett. A 5677

[17] Hamity V and Lamberti W 1987 A family of rotating disks as sources of the Kerr metric Gen. Rel. Grav. 19917

[18] Keres H 1967 Physical interpretation of solutions to the Einstein equations Zh. Eksper. Teor. Fiz. 52768

[19] Lopez C A 1981 Rigidly rotating disk as a source of the Kerr geometry Nuovo Cimento B 6617

[20] Morgan T and Morgan L 1969 The gravitational field of a disc Phys. Rev. 1831097

[21] Bičák J, Lynden-Bell D and Katz J 1993 Relativistic disks as sources of static vacuum spacetimes Phys. Rev. D 474334

[22] Bičák J and Ledvinka T 1993 Relativistic disks as sources of the Kerr metric Phys. Rev. Lett. 711669

[23] Poisson E 2004 A Relativists Toolkit (Cambridge: Cambridge University Press) pp 90-5

[24] Haggag S and Marek J 1981 A nearly perfect fluid source for the Kerr metric Nuovo Cimento B 62273

[25] Haggag S 1990 A fluid source for the Kerr metric Nuovo Cimento B 1054

[26] Ramadan M A 2004 Fluid sources for the Kerr metric Nuovo Cimento B 119123

[27] Smith N, Hinkle K H and Ryde N 2008 Red supergiants as potential type IIn supernova progenitors: spatially resolved 4.6 micron CO emission around VY CMa and Betelgeuse arXiv/astro-ph/0811.3037

[28] Turnball A J, Bridges T J and Carter D 1999 Imaging of the shell galaxies NGC474 and NGC7600 and implications for their formation Mon. Not. R. Astron. Soc. 304967

[29] Gill C D and O'Brien T J 2000 Hubble space telescope imaging and ground-based spectroscopy of old nova shells: I. FH Ser,V533 Her, BT Mon, DK Lac, V476 Cyg Mon. Not. R. Astron. Soc. 314175 
[30] Canalizo G, Bennert N, Jungwiert B, Stockton A, Schweizer F, Lacy M and Peng C 2007 Spectacular shells in the host galaxy of the QSO MC2 1635+119 Astrophys. J. 669201

[31] Misner C W, Thorne K S and Wheeler J A 1970 Gravitation (San Francisco: Freeman) p 894

[32] Carroll S 2004 Spacetime and Geometry (New York: Addison-Wesley) p 254

[33] Glass E N and Krisch J P 2004 Spinning up asymptotically flat spacetimes Class. Quantum Grav. 215543

[34] Ansorg M and Petroff D 2006 Negative Komar mass of single objects in regular, asymptotically flat spacetimes Class. Quantum Grav. 23 L81

[35] Ansorg M and Petroff D 2007 Negative Komar masses in regular stationary spacetimes arXiv/gr-qc/0708.3899

[36] Balasin H and Nachbagauer H 1994 Distributional energy-momentum tensor of the Kerr-Newman spacetime family Class. Quantum Grav. 111453 